\section{Endoplasmic reticulum stress controls iron metabolism through TMPRSS6 repression and hepcidin mRNA stabilization by RNA-binding protein HuR}

The liver hormone hepcidin controls the main inflows of iron into plasma by binding to and inducing the degradation or the occlusion of the iron export activity of ferroportin, the only known cellular exporter of iron., When hepcidin concentrations are high, iron is trapped in enterocytes of the duodenum, hepatocytes, and macrophages. Hepcidin production by the hepatocytes is induced by a number of stimuli, most notably iron, through the BMP-SMAD signaling pathway, ${ }^{3}$ and inflammatory signals, through the IL-6/ STAT3 signaling axis. ${ }^{4}$ In addition, hepcidin has also been reported to respond to intracellular stress, namely endoplasmic reticulum (ER) stress which is involved in a number of pathophysiological states, including the inflammatory response, nutrient disorders and viral infection. A previous study has suggested that hepcidin induction by ER stress is controlled by the BMP-SMAD pathway, ${ }^{5}$ but the exact mechanism is still uncertain.

ER stress may have an important role in the development of nonalcoholic fatty liver disease (NAFLD). Indeed, hepatic lipid accumulation induces ER stress, and, in turn, the ER stress response promotes hepatic lipogenesis, thus creating a positive-feedback loop, which may contribute to the development of hepatic steatosis. ${ }^{6}$ ER stress has also been implicated in the development of hepatocellular injury and fibrosis and the progression of simple steatosis to nonalcoholic steatohepatitis (NASH). Interestingly, approximately one third of patients with NAFLD show signs of disturbed iron homeostasis as indicated by elevated serum ferritin with normal or mildly elevated transferrin saturation, mild hepatic iron deposition $^{7}$ and increased hepcidin production. ${ }^{8}$ Excess iron is proposed to aggravate the natural course of NAFLD because of its capability to catalyze the formation of toxic hydroxyl radicals that cause cellular damage. Iron accumulation in NAFLD is mainly due to impaired iron export from hepatocytes and Kupffer cells ${ }^{7}$ which might well be the consequence of hepcidin induction by ER stress.

Given the potential impact of ER stress-induced hepcidin on hepatic iron deposition in NAFLD patients, it is essential to better understand the molecular mechanisms leading to the induction of hepcidin in this context. In order to definitely elucidate these mechanisms, we used a model of acute ER stress induced in mice by tunicamycin. We demonstrated that induction of hepcidin by ER stress requires repression of TMPRSS6, the gene coding for the inhibitor of BMP-SMAD1/5/8 signaling, matriptase-2, and stabilization of hepcidin mRNA by the RNA-binding protein, HuR.

In order to investigate the kinetics of hepcidin induction by ER stress over time, wild-type (WT) mice received one intraperitoneal (IP) injection of tunicamycin (Tm) (2 $\mathrm{mg} / \mathrm{kg}$ ), a well-known ER stress inducer, and were sacrificed at time points ranging from 3 to 24 hours after injection. As expected, Tm injection triggered ER stress in the liver (Online Supplementary Figures $S 1 A-B, S 2 A-B$ ). As shown in Online Supplementary Figure $S 1 C$, hepcidin gene expression progressively increased and reached a maximum 6 hours after Tm injection. Therefore, this time point was chosen for performing all the following experiments.

As expected, we show that hepcidin induction coincides with an activation of the BMP-SMAD pathway
(Figure 1A-C; Online Supplementary Figure S1D) which is dependent on the Bmp type 1 receptor Bmpr1a (Online Supplementary Figure S3) 6 hours after Tm injection. In this study, our objective was to find out the mechanisms that activate BMP-SMAD signaling during ER stress, leading to excessive hepcidin production. As shown in the Online Supplementary Figure $S 4 A-H$, Tm did not increase the expression of the ligands known to activate $\mathrm{Bmp}-\mathrm{Smad}$ signaling and hepcidin gene expression in circumstances other than ER stress, i.e., Bmp6 ${ }^{3}$ and $\mathrm{Bmp} 2^{9}$ and does not modulate the expression of other genes belonging to this pathway. Another ligand of the TGF- $\beta$ family, activin B, was suggested to induce hepcidin in ER stress5 but, as shown in the Online Supplementary Figure S4I-J, Tm similarly induced IdI and hepcidin gene expression in Inhbb ${ }^{-/}$mice, lacking activin B, and in WT controls. A role for activin B in this process is thus unlikely.

Matriptase-2, encoded by the TMPRSS6 gene, is a strong inhibitor of the BMP-SMAD signaling pathway and of hepcidin expression..$^{10}$ Interestingly, Tm injection significantly suppressed matriptase-2 at mRNA (Figure 1D; Online Supplementary Figure S1E) and protein (Online Supplementary Figure S5) levels, which could explain the observed activation of BMP-SMAD signaling and induction of hepcidin expression in these mice. In order to confirm this hypothesis, we assessed the response of Tmprss $6^{-/}$mice to Tm. Notably, in the absence of stimulation, Smad5 phosphorylation and Id 1 expression are, as expected, constitutively high in Tmprss $6^{--}$mice, but they have not reached their peak and can still be further induced by iron dextran (Online Supplementary Figure S6FG). This demonstrates that Tmprss $6^{-1-}$ mice have the ability to activate the BMP-SMAD signaling in response to external stimuli. However, although ER stress was similarly induced in both Tmprss6 $6^{-1}$ and WT mice (Online Supplementary Figure S6A-D), Smad5 phosphorylation (Figure $1 \mathrm{E}$ ) and $I d 1$ expression (Figure $1 \mathrm{~F}$ ) were not further increased by Tm in Tmprss $6^{-1}$ mice. In order to determine if the loss of BMP-SMAD activation was not blunted by the iron deficiency anemia of Tmprss $6^{-1 /}$ mice, we used $\mathrm{Bmpb}^{-1-}$ - Tmprss $6^{-/}$mice which have a BMP signaling similar to WT mice and no iron deficiency anemia. ${ }^{11}$ In this mouse model, BMP signaling is not induced by Tm injection either (Online Supplementary Figure S7A-B) confirming that repression of matriptase- 2 is required for activation of BMP-SMAD signaling by ER stress. Of note, lack of Bmp6 only does not prevent the induction of BMP signaling and hepcidin expression in response to ER stress (Online Supplementary Figure S8).

Quite surprisingly though, and despite the lack of further Smad5 activation, hepcidin induction was not totally abolished in mice lacking matriptase-2 (Figure 1G; Online Supplementary Figure S7C), suggesting that a second mechanism contributes to the whole magnitude of hepcidin upregulation in ER stress.

In order to characterize this additional mechanism observed in Tmprss6 $6^{-1}$ mice, we used the HepG2 hepatoma cell line that expresses TMPRSS6 mRNA at a level so low that a siRNA directed against it is unable to promote any activation of BMP-SMAD signaling (data not shown). The HepG2 cell line is thus a good model to characterize hepcidin regulation by ER stress independently of matriptase-2 and BMP-SMAD signaling. Treatment of HepG2 cells with Tm induces ER stress (Figure 2A) and hepcidin (Figure 2B; Online Supplementary Figure $S 9 A$ ) even in the absence of Smad5 activation or ID1 mRNA induction (Figures 5C-D; Online Supplementary Figure S9B). 
The cytoplasmic level of a messenger RNA relies not only on its rate of synthesis but also on its decay rate. Therefore, in order to determine if the hepcidin level in response to $\mathrm{Tm}$ is regulated through induction of its transcription or through increased stability of its mRNA, HepG2 cells were treated with the transcriptional inhibitor actinomycin D. As expected, treatment with actinomycin D significantly reduced hepcidin mRNA lev- els in these cells (Figure 2E). However, when treated with Tm in the presence of actinomycin D, HepG2 cells still exhibited a significant increase in hepcidin mRNA expression (Figure 2E), clearly demonstrating that ER stress controls hepcidin gene expression through posttranscriptional mechanisms.

Interestingly, ELAVL1/HuR was recently described as a protein stabilizing hepcidin mRNA in response to satu-
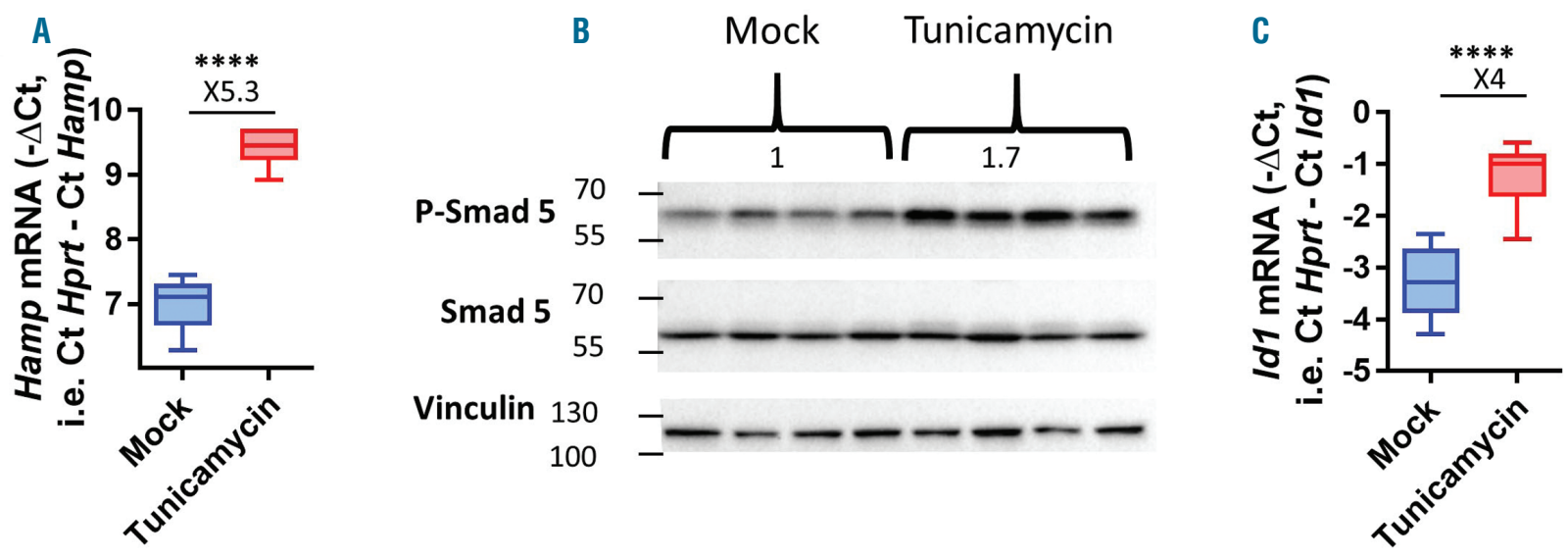

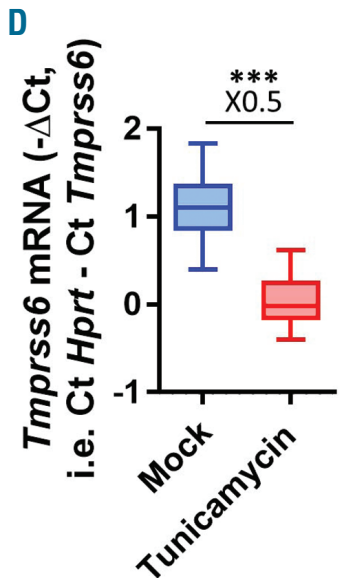

F

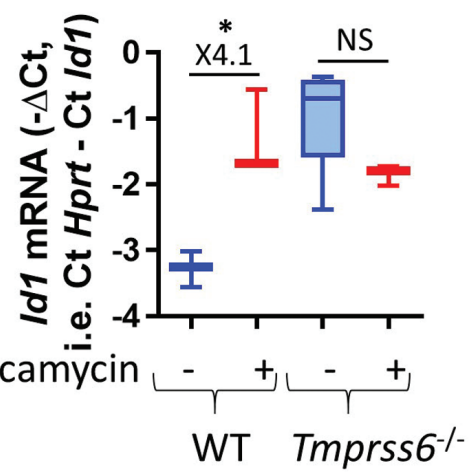

$\mathbf{E}$

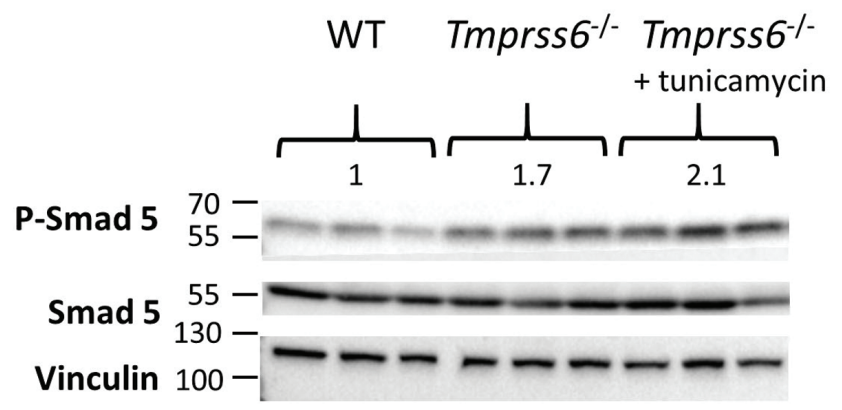

G

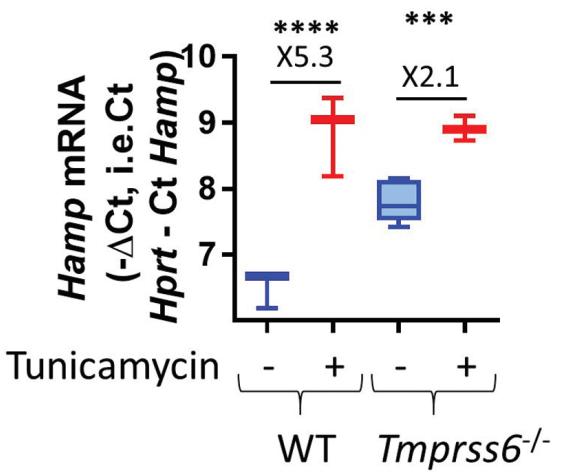

Figure 1. Endoplasmic reticulum stress upregulates hepcidin expression and activates the BMP-SMAD signaling pathway partially through matriptase-2. Wildtype (WT) CD1 mice (6-9/group) were injected with mock (blue boxes) or tunicamycin (red boxes) and were analyzed 6 hours (h) later for liver (A) Hamp mRNA expression; (B) P-Smad 5 relative to total Smad5 protein expression; (C) Id1 mRNA expression and (D) Tmprss 6 mRNA expression. C57BI/6 WT mice and Tmprss6 ${ }^{-/}$mice (3-4/group) were injected with mock (blue boxes) or tunicamycin (red boxes) and were analyzed $6 \mathrm{~h}$ later for liver (E) P-Smad 5 relative to total Smad 5 protein expression; (F) Id1 mRNA expression and (G) Hamp mRNA expression. Estimates of the fold changes in gene expression ( $2^{-\Delta \Delta c t}$ ) are shown on the graphs. $* P<0.05 ; * * * P<0.001 ; * * * * P<0.0001$. 
rated fatty acids. It acts through a direct interaction with the AU-rich elements (ARE) located in the 3'UTR of hepcidin mRNA. ${ }^{12}$ Importanly, we observed that HuR mRNA expression is increased in the liver in response to $\mathrm{Tm}$ treatment (Online Supplementary Figure S1F). HuR was thus a good candidate for the control of hepcidin mRNA stability not only in response to fatty acids but also to ER stress. In HepG2 cells transfected with a siRNA pool directed against human $\mathrm{HuR}$, HuR silencing reduced $H u R$ mRNA expression (Figure $2 \mathrm{~F}$ ) and, prevented a significant increase of hepcidin mRNA in response to Tm (Figure $2 \mathrm{G})$. In order to determine if, in response to Tm, HuR regulates hepcidin mRNA stability through direct binding to hepcidin 3'UTR, we performed RNA-CLIP experiments with HuR or IgG control antibodies followed by quantitative RT-PCR with primers specific for the hep-
A

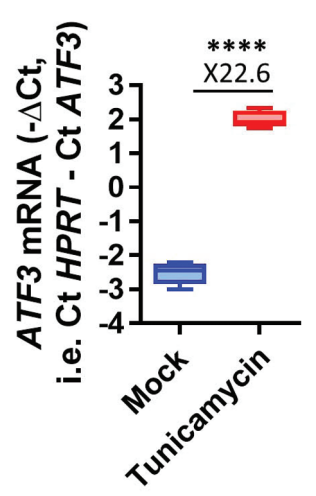

D

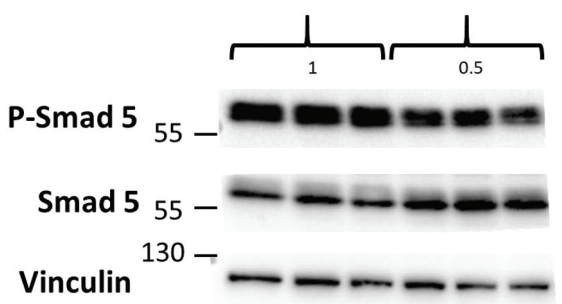

B

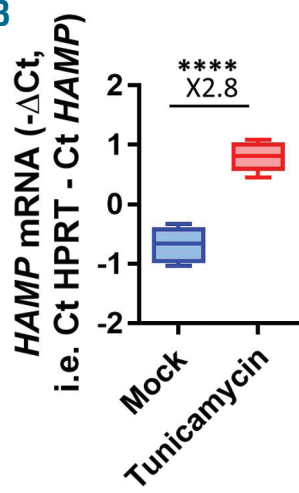

$\mathrm{E}$

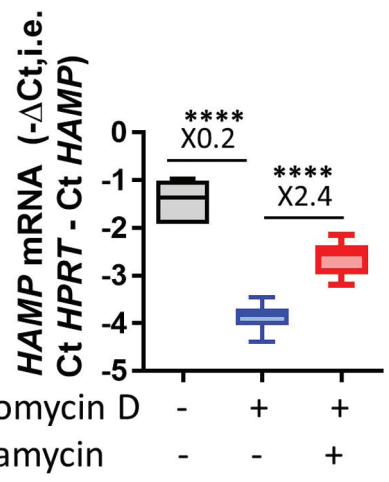

H

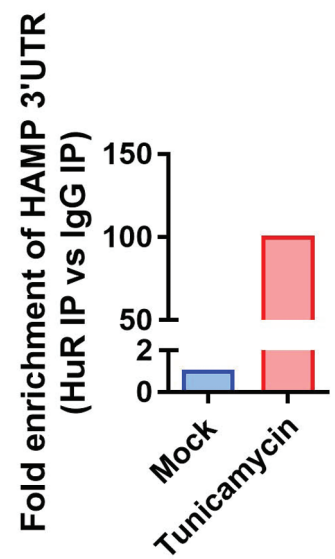

C

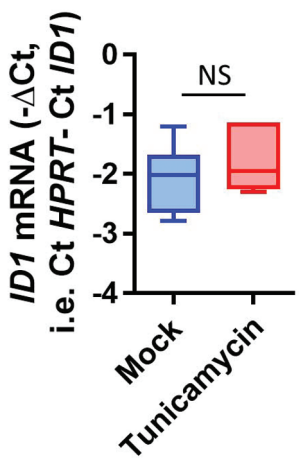

$\mathbf{F}$

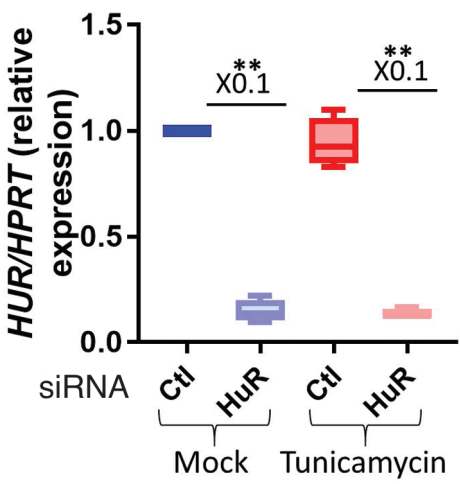

I

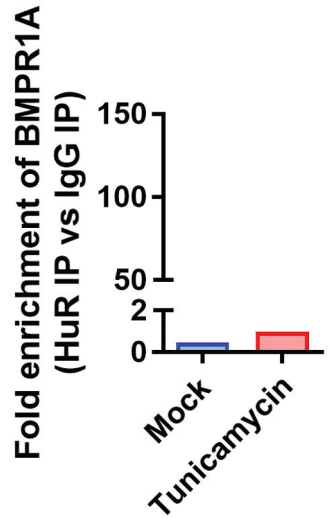

Figure 2. Endoplasmic reticulum stress stabilizes hepcidin mRNA through HuR in vitro. HepG2 cells were treated with mock (blue boxes) or tunicamycin (Tm) (red boxes) and analyzed for (A) ATF3 mRNA expression; (B) HAMP mRNA expression; (C) ID1 mRNA expression and (D) P-Smad 5 relative to total Smad 5 protein expression. Values shown are the result of three independent experiments performed in duplicate. (E) HepG2 cells were treated with mock (black box) or actinomycin D alone (blue box) or together with Tm (red box) for 6 hours (h) and analyzed for HAMP mRNA expression. (F-G) HepG2 cells were transfected either with a control pool of small interfering RNA (siRNA) or a pool of siRNA designed to silence human HuR and subjected to either treatment with mock (blue box) or Tm (red box) for $6 \mathrm{~h}$. The results of four experiments were analyzed by repeated measures one-way ANOVA. Fold-change compared to cells transfected with siRNA control (ctl) and treated with mock are shown on the graphs. Total lysates from HepG2 cells treated with mock or Tm were subjected to cross-linking immunoprecipitation CLIP-seq RNA-CLIP with either HuR or control normal IgG antibodies. RNA was collected from the immunoprecipitates and analyzed for (H) HAMP 3'untranslated region (UTR) sequence and (I) a non-relevant sequence (BMPR1A) enrichment by quantitative RT-PCR. One representative experiment over three independent experiments is presented. Estimates of the fold changes in gene expression $\left(2^{-\Delta \Delta c t}\right)$ are shown on the graphs. ${ }^{\star} P<0.05$; $\star \star P<0.01$; $* * * * P<0.0001$. NS: not sigificant. 
cidin 3'UTR sequence or a non-relevant sequence in the $B M P R 1 A$ gene. In cells treated with $\mathrm{Tm}$, the level of hepcidin 3'UTR measured in the immunoprecipitate collected with HuR antibody was increased 20 to 6,700-fold relative to that in the immunoprecipitate collected with control IgG (Figure 2H; Online Supplementary Figure S10A$B)$. No such enrichment of hepcidin 3'UTR is seen in cells treated with mock. Furthermore, there was no enrichment of the non-relevant sequence in the immunoprecipitate collected with $\mathrm{HuR}$ antibody (Figure 2I; Online Supplementary Figure S10C-D), indicating that Tm treatment specifically promotes binding of the RNA stabilizer HuR to hepcidin 3'UTR.

Interestingly, while HuR mRNA expression is stimulated in response to ER stress in WT and Tmprss6 $6^{-1-}$ mice (Online Supplementary Figures S1F, S6E), it is blunted when Bmpr1A is missing (Online Supplementary Figure $\mathrm{S} 3 \mathrm{H})$. This is consistent with the previously reported transcriptional control of HuR by BMP-SMAD signaling. ${ }^{13}$ Accordingly, hepcidin mRNA expression is not stabilized in Bmpr1 $A^{\text {hephep }}$ mice.

In the present study, we showed that ER stress up-regulates the iron-regulatory hormone hepcidin through two complementary mechanisms. The first involves the inhibition of matriptase-2, which activates BMP-SMAD signaling without the induction of Bmp2 and Bmp6 or the requirement for activin $B$. The second is the stabilization of hepcidin mRNA by the RNA-binding protein, HuR. These two mechanisms appear to act sequentially. Indeed, whereas the induction of another target of BMP-SMAD signaling, Id1, reached a plateau 3 hours after Tm injection (Online Supplementary Figure S1D), hepcidin mRNA did not achieve its maximal level at this time point and continued to rise at least 2 -fold between 3 and 6 hours (Online Supplementary Figure S1C). This coincided with the induction of Hur expression in the liver (Online Supplementary Figure S1F). Thus, the effect of $\mathrm{HuR}$ on hepcidin mRNA stabilization is very likely secondary to the activation of BMP-SMAD signaling. Moreover, in WT mice, in which both mechanisms are functional, hepcidin is increased 5.3 times whereas in $\mathrm{Bmp6}^{-1-}$-Tmprss $6^{-1-}$ mice in which the BMP signaling is not activated, hepcidin expression is only induced 2.8 times. This suggests that both mechanisms each contribute to half of the whole magnitude of hepcidin induction in response to ER stress. Interestingly, $H u R$ mRNA is not increased in mice lacking $B m p r 1 A$ specifically in hepatocytes. A functional BMP-SMAD signaling pathway is thus necessary for the induction of hepcidin by ER stress.

Chronic liver ER stress, promoted at least in part by liver fatty acid accumulation, ${ }^{6}$ contributes to the progression of NAFLD towards NASH. ${ }^{14-16}$ Mild to moderate hepatic iron accumulation, seen in hepatocytes and/or in cells of the reticuloendothelial system of one third of the patients with NAFLD, ${ }^{7}$ is also a factor of poor prognosis. Finally, NAFLD patients often have high levels of hepcidin. In vitro, HuR stabilizes hepcidin mRNA in response not only to ER stress as demonstrated here but also to saturated fatty acids. ${ }^{12}$ As saturated fatty acids accumulate in the liver in NAFLD and induce ER stress, it is highly likely that ER stress is the intermediary between fatty acids and activation of $\mathrm{HuR}$ and that $\mathrm{HuR}$ also participates in hepcidin regulation in patients with NAFLD. Nonetheless, the role of HuR on the stabilization of hepcidin in vivo will need to be confirmed by further studies.

Audrey Belot, ${ }^{1}$ Ophélie Gourbeyre, ${ }^{1}$ Anais Palin, ${ }^{1}$ Aude Rubio, ${ }^{1}$ Amélie Largounez, ${ }_{1}^{1}$ Céline Besson-Fournier, ${ }^{1}$ Chloé Latour, ${ }^{1}$ Megane Lorgouilloux, ${ }_{1}^{1}$ Inka Gallitz, ${ }^{2}$
Alexandra Montagner, ${ }^{3}$ Arnaud Polizzi, ${ }^{3}$ Marion Régnier, ${ }^{3}$ Sarra Smati, ${ }^{3}$ An-Sheng Zhang, ${ }^{4}$ Manuel D. Diaz-Munoz, Andrea U. Steinbicker, ${ }^{2}$ Hervé Guillou, ${ }^{3}$ Marie-Paule Roth, ${ }^{1}$ Hélène Coppin ${ }^{1}$ and Delphine Meynard"

${ }^{1} I R S D$, Université de Toulouse, INSERM, INRA, ENVT, UPS, Toulouse, France; ${ }^{2}$ Department of Anesthesiology, Intensive Care and Pain Medicine, University Hospital Muenster, University of Muenster, Muenster, Germany; Institut National de La Recherche Agronomique (INRA), UMR1331 ToxAlim, Toulouse, France and

${ }^{4}$ Cell, Developmental, and Cancer Biology, Oregon Health \& Science University, Portland, OR, USA and ${ }^{5}$ CPTP, INSERM

UMR1043/CNRS U5282, Toulouse, France

Correspondence:

DELPHINE MEYNARD - delphine.meynard@inserm.fr

doi:10.3324/haematol.2019.237321

Disclosures: no conflicts of interest to disclose.

Contributions: $A B, O G, A P, A R$, and $A L$ performed experiments, analyzed, and discussed results; $C B-F, C L, M L, I G, A M, A P, M R$, $S S$ and AUS performed experiments; MD-M, AUS, HG analyzed and discussed results; $A-S Z$ provided antibody against matriptase-2, $D M, H C$, and M-PR discussed data and wrote the manuscript; $D M$ designed research, performed experiments, analyzed and discussed data, and wrote the manuscript; and all authors reviewed and approved the final manuscript.

Acknowledgments: the authors are grateful to Carlos Lopez Otin (University of Oviedo, Oviedo, Spain) for kindly providing the original Tmprss6 $6^{-1-}$ mice on a mixed genetic background. They also thank Florence Capilla (Experimental Histopathology Platform, Toulouse Purpan), and members of the INSERM USO06 facility (Toulouse) for their technical assistance and help in the mouse breeding.

Funding: DM was supported by the Cooley's Anemia Foundation, the French Foundation for Rare Diseases, the Région Midi-Pyrénées and ANR (ANR-17-CE14-0036-01 and ANR-17-CE14-0031-01). AUS was supported by a research grant of the German Research Foundation (DFG, STE-1985/4-1). HG was supported by grants from Région Occitanie and ANR (ANR-15-CE14-0026-Hepatokind). MDD- $M$ was supported by ATIP-AVENIR (INSERM/CNRS) program and by Plan-Cancer (C18003BS). A-SZ was supported by a grant from NIH (R01DK102791). This work was also supported by the "Programme des Investissements d'Avenir" ANINFIMIP (ANR-11EQPX-0003).

\section{References}

1. Nemeth E, Tuttle MS, Powelson J, et al. Hepcidin regulates cellular iron efflux by binding to ferroportin and inducing its internalization. Science. 2004;306(5704):2090-2093.

2. Aschemeyer S, Qiao B, Stefanova D, et al. Structure-function analysis of ferroportin defines the binding site and an alternative mechanism of action of hepcidin. Blood. 2018;131(8):899-910.

3. Meynard D, Kautz L, Darnaud V, Canonne-Hergaux F, Coppin H, Roth MP. Lack of the bone morphogenetic protein BMP6 induces massive iron overload. Nat Genet. 2009;41(4):478-481.

4. Nemeth E, Rivera S, Gabayan V, et al. IL-6 mediates hypoferremia of inflammation by inducing the synthesis of the iron regulatory hormone hepcidin. J Clin Invest. 2004;113(9):1271-1276.

5. Canali S, Vecchi C, Garuti C, Montosi G, Babitt JL, Pietrangelo A. The SMAD pathway is required for hepcidin response during endoplasmic reticulum stress. Endocrinology. 2016;157(10):3935-3945.

6. Wang D, Wei Y, Pagliassotti MJ. Saturated fatty acids promote endoplasmic reticulum stress and liver injury in rats with hepatic steatosis. Endocrinology. 2006;147(2):943-951

7. Nelson JE, Wilson L, Brunt EM, et al. Relationship between the pattern of hepatic iron deposition and histological severity in nonalcoholic fatty liver disease. Hepatology. 2011:53(2):448-457.

8. Nelson JE, Klintworth H, Kowdley KV. Iron metabolism in Nonalcoholic Fatty Liver Disease. Curr Gastroenterol Rep. 2012; 14(1):8-16.

9. Koch PS, Olsavszky V, Ulbrich F, et al. Angiocrine Bmp2 signaling in murine liver controls normal iron homeostasis. Blood. 2017; 
129(4):415-419.

10. Du X, She E, Gelbart T, et al. The serine protease TMPRSS6 is required to sense iron deficiency. Science. 2008;320(5879):1088-1092.

11. Nai A, Rubio A, Campanella A, et al. Limiting hepatic Bmp-Smad signaling by matriptase-2 is required for erythropoietin-mediated hepcidin suppression in mice. Blood. 2016;127(19):2327-2336.

12.Lu S, Mott JL, Harrison-Findik DD. Saturated fatty acids induce posttranscriptional regulation of HAMP mRNA via AU-rich elementbinding protein, human antigen R (HuR). J Biol Chem. 2015 290(40):24178-24189.

13.Jeyaraj SC, Singh M, Ayupova DA, Govindaraju S, Lee BS Transcriptional control of human antigen $\mathrm{R}$ by bone morphogenetic protein. J Biol Chem. 2010 2;285(7):4432-4440.

14. Kammoun HL, Chabanon H, Hainault I, et al. GRP78 expression inhibits insulin and ER stress-induced SREBP-1c activation and reduces hepatic steatosis in mice. J Clin Invest. 2009;119(5):12011215

15. Oyadomari S, Harding HP, Zhang Y, Oyadomari M, Ron D. Dephosphorylation of translation initiation factor 2alpha enhances glucose tolerance and attenuates hepatosteatosis in mice. Cell Metab. 2008;7(6):520-532

16. Xiao G, Zhang T, Yu S, et al. ATF4 protein deficiency protects against high fructose-induced hypertriglyceridemia in mice. J Biol Chem. 2013;288(35):25350-25361. 